\title{
Geostatistical Estimation of Electromagnetic Exposure
}

\author{
Y. O. Isselmou, H. Wackernagel, W. Tabbara and J. Wiart
}

\begin{abstract}
The electromagnetic environment in urban areas is growing increasingly complex. Sources of electromagnetic exposure like TV, FM, GSM, Wifi and others are spreading continuously and in the case of Wifi their geographical locations cannot be cataloged exhaustively anymore. Furthermore, the complexity of any highly urbanized environment and the lack of information about the dielectric properties of buildings lead to complex configuration so that a precise deterministic modeling of the electromagnetic exposure at any a given location of interest is probably out-of-reach.

On the other hand there is a growing demand to assess the human exposure induced by these wireless communications. In a project between France Télécom R \& D, Ecole des Mines and Supélec the application of geostatistical methods in this context is being explored.

Geostatistics provides the right framework for setting up such exposure maps and its spatial statistical model yields an estimate of exposure as well as an associated error (De Doncker et al., 2006).

The project consists of three phases: geostatistical evaluation of data generated by the numerical model EMF Visual (both in free space and with the addition of obstacles), statistical analysis of measurements performed in the area of the Quartier Latin in Paris and, finally, joint evaluation of an urban area both by statistical and deterministic numerical modeling.

The paper reports about the third phase of this ongoing project, in which the spatial variation is modeled using the variogram, followed by a spatial regression known as kriging. The paper presents results about using a kriging algorithm that integrates numerical model output as an external drift.
\end{abstract}

Y. O. Isselmou

France Télécom R \& D, RESA/FACE, Issy-les-Moulineaux, France 


\section{Introduction}

The wireless communication systems and cellular handset are nowadays intensively used worldwide. To support these equipments thousand of base stations or access points operating at different frequencies and using different communication protocol have been implemented. These antennas have induced a public concern about exposure to electromagnetic fields. To check the compliance with the relevant limits such as those of ICNIRP (1998) international bodies such as CENELEC, IEC, CEPT or IEEE have developed accurate methods to assess exposure. The exposure to the electromagnetic field induced by TV, GSM, FM... is weak as shown in ANFR (2004), yet the public is looking for the exposure assessment and not only for the compliance. Numerical tools such as EMF Visual have been developed, but even if the locations of the antennas are public (e.g. www.cartoradio.fr or www.sitefinder.radio.gov.uk) the characteristics of emission (type of antenna, tilt, azimuth, power emitted) are considered as quite sensitive data. Because of that, simulations are not always possible, and an exposure assessment based on field measurements is needed. A frequency selective "in situ" exposure assessment can be carried out using either a personal dosimeter or a monitoring station (e.g. EME SPY and INSITE Station by Antennessa), but tools have to be developed to spatially interpolate the data.

In this paper we propose a new approach to mapping electromagnetic exposure which resembles methods that are common practice in numerical weather forecasting (Daley, 1991). The approach is subdivided into two main steps.

First, a guess of the electromagnetic field is constructed by a numerical model, using the known antenna positions as well as likely parameters for their emission characteristics. This provides a possible, yet inaccurate first picture of the propagation of the electromagnetic signal in the field.

Second, the surface generated with the numerical model, the guess field, is combined with the measurements of radio-electric exposure to base station emissions in order to provide a corrected picture of the electromagnetic field. The combination of the data with the numerical model output is performed using the geostatistical method of kriging, which is preceded by a statistical analysis and modeling of the residual between the guess field and the observations.

\section{Electromagnetic Field Strength Assessment}

The wireless communications services operate at different frequencies, for instance the frequency band used by FM, TV3, GSM 900 downlink, GSM 1800 downlink, UMTS downlink and WIFI are respectively in the MHZ [88 108], [174 223], [925 960], [1805 1880], [2110 2170], [2400 2500] bands. The wireless systems use also different communication protocols and techniques (Wiart et al., 2000) such as Time Division Multiplex Access (TDMA), Discontinuous Transmission (DTX), Code Division Multiplex Access (CDMA) and Adaptive Power Control (APC), therefore the power emitted by these systems are variable. Moreover, the signal emitted by 


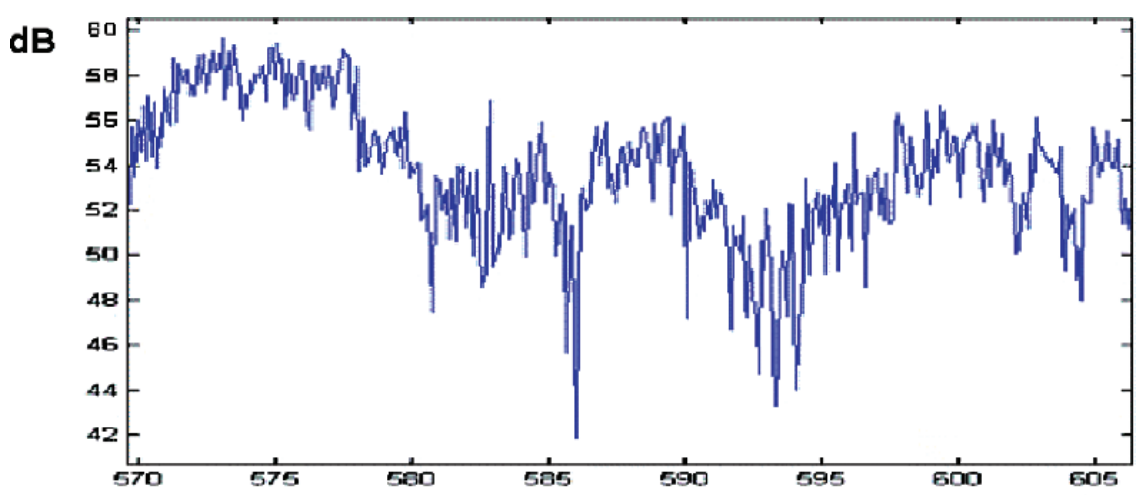

Fig. 1 Electric field strength $(\mathrm{dB} \mu \mathrm{v} / \mathrm{m})$ along a line

an antenna operating at a given frequency is reflected and diffracted by walls and edges of buildings. Therefore the field received is a combination in phase and in counter-phase of these contributions, which create fading (see Fig. 1)

The exposure assessment has to take into account such variations (Larchevêque et al., 2005) and measurements performed at different locations cannot be compared point to point.

\section{Measurement System}

Different frequency-selective measurement systems exist: on the one hand, integrated equipments based on hard filters such as the personal dosimeter, on the other hand, the classical spectrum analyzer. These equipments are in either case connected to an antenna.

In the case of a personal dosimeter the frequency bands under test are predetermined and consist of the main services occupying the spectrum, such as TV, FM, GSM, UMTS, WIFI. The dosimeter made available for this study (see Fig. 2) uses hardware filters and was able to measure 9 bands (a newer version will be able to analyze 12 bands). This included GSM 900, GSM 1800 and UMTS downlink (i.e emitted by base stations). The axial isotropy of the device is greater than $1 \mathrm{~dB}$ at $66 \%$ and greater than $2 \mathrm{~dB}$ at $95 \%$; the minimum and maximum detection is respectively $0.05 \mathrm{~V} / \mathrm{m}$ and $5 \mathrm{~V} / \mathrm{m}$; the resolution is $0.01 \mathrm{~V} / \mathrm{m}$.

The memory of the dosimeter is able to record up to 7000 measurements with a minimum sampling duration of 3 seconds. Figure 3 shows a set of 220 measurements performed along streets in Paris with a sampling duration of 10 seconds. Since the sensitivity of the equipment is $0.05 \mathrm{~V} / \mathrm{m}$, the field strength below or equal to this limit is recorded at 0.05 and is not significant.

The spectrum analyzer is a versatile equipment: its output consists in a trace that represents the spectrum sampled at $N$ points in a frequency interval ( $f$ start, 


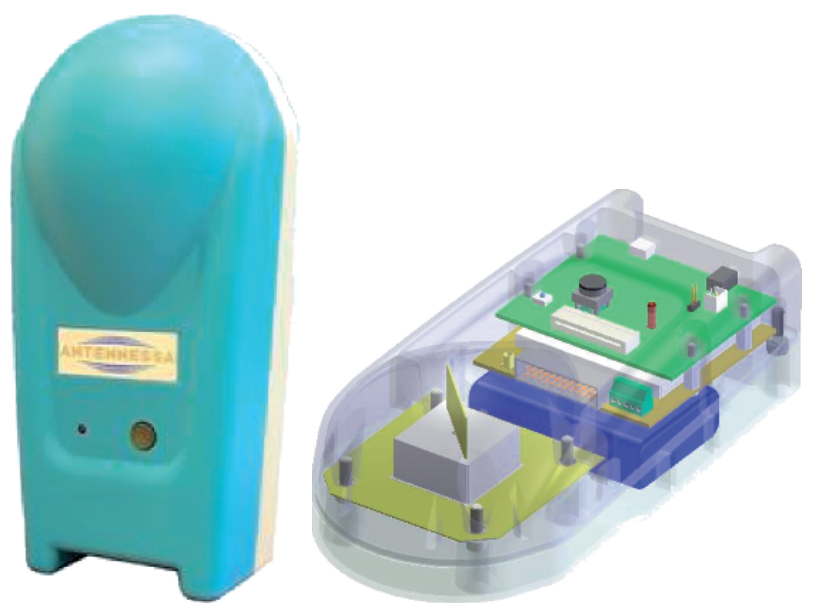

Fig. 2 View of the dosimeter used in this study and its internal structure

fstop) that can be defined as needed. The spectrum analyzer is more sensitive and its resolution is smaller, but the equipment is less portable.

Since measurements can be performed with both methods we have carried out a comparison between spectrum analyzer and dosimeter measurements. This study

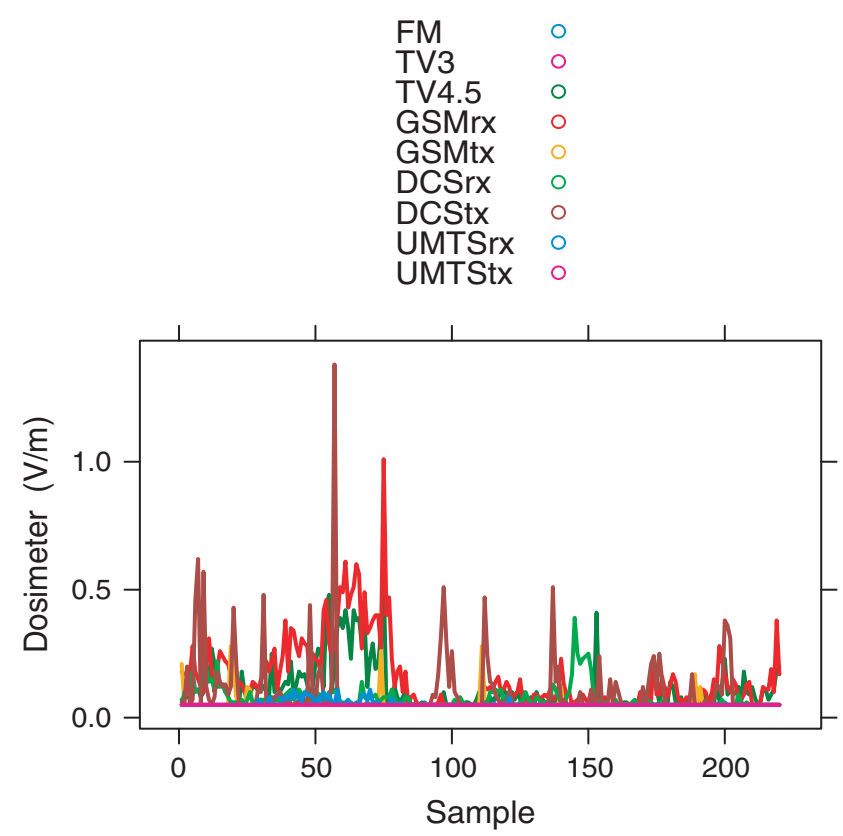

Fig. 3 Example of 220 measurements (sampling duration 10s) performed with the dosimeter along streets in the Quartier Latin in Paris 


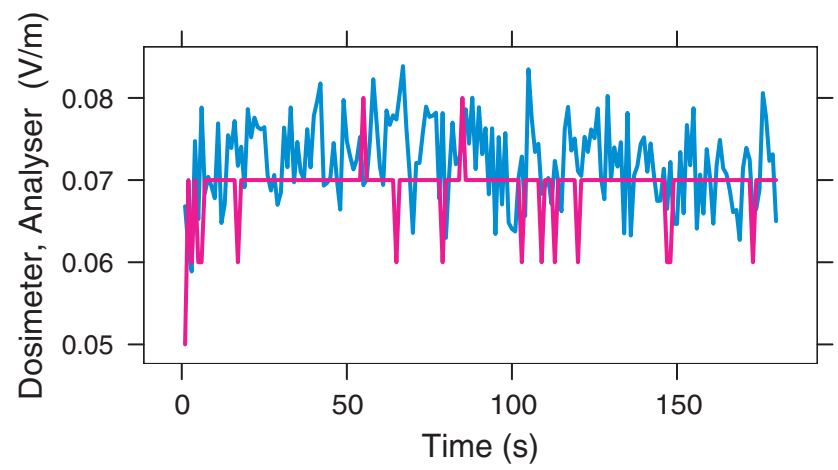

Fig. 4 Comparisons between measurement performed in laboratory, at $900 \mathrm{MHz}$, with a dosimeter and a spectrum analyzer

has been performed in laboratory and has been focused on GSM 900 downlink, known as GSM900rx, since this will be the frequency of interest in this study. The Fig. 4 reveals that there is a good agreement between the measurements from the two devices. As explained previously, the measurements performed using a spectrum analyzer are more precise, so the measurements exhibit only a small fading. The measurements from the dosimeter have a limited resolution as compared to the spectrum analyzer. The comparison further shows that measurements performed with a dosimeter match well those stemming from the spectrum analyzer.

\section{Data from the Quartier Latin and EMF Visual Simulation}

The first author took sample dosimeter measurements in the Quartier Latin area of Paris. The sequence of the measurements for the different frequency bands was displayed on Fig. 3. We will concentrate on the GSMrx band, which corresponds to the radio-electric exposure related to emissions from GSM base stations.

The histogram of the 220 GSMrx values is displayed on Fig. 5 and it has a typical right-skew shape. The tail has been colored in blue and it can be noted that three values single out on the right, which we may qualify as outliers.

The geographical locations of the 220 samples can be seen on Fig. 6. The symbols are proportional in size to the values of GSMrx exposure measured at each location. We note that two of the three outliers are located next to very low values so that we may expect strong small-scale spatial variation.

Taking account of the base station locations and of the reflectors (buildings) in that section of Paris, as displayed on Fig. 7, a simulation of electromagnetic propagation was performed with the software EMF Visual. The geographical map of the output is shown on Fig. 8 and the corresponding histogram on Fig. 9. As the characteristics of the antennas were not disclosed to us, the parameters were set to likely values and we 


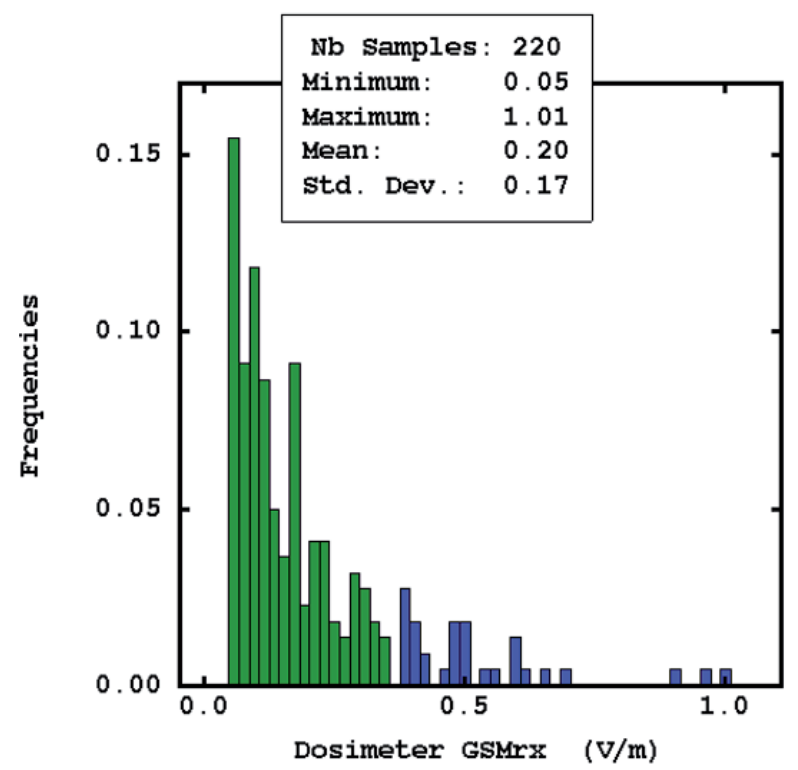

Fig. 5 Histogram of dosimeter GSMrx data; There are 3 outliers on the right

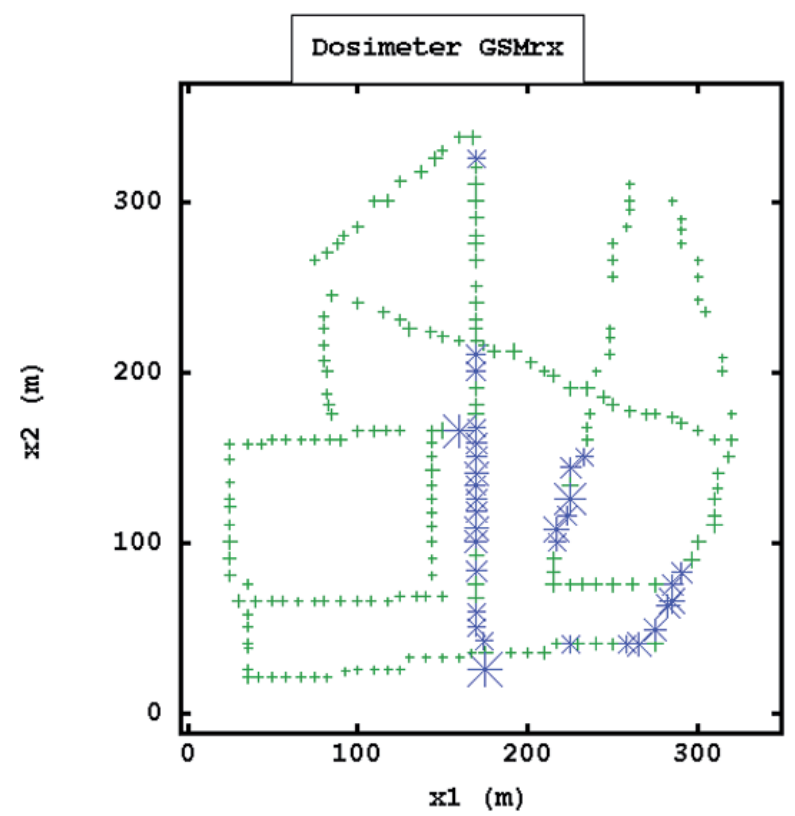

Fig. 6 Map of sampling locations of dosimeter GSMrx data in the Quartier Latin; the diagonal line of samples was taken along boulevard Saint Germain. The stars correspond to values in the tail of the histogram; the symbol sizes are proportional to the values. Two of the 3 outliers are next to low values 

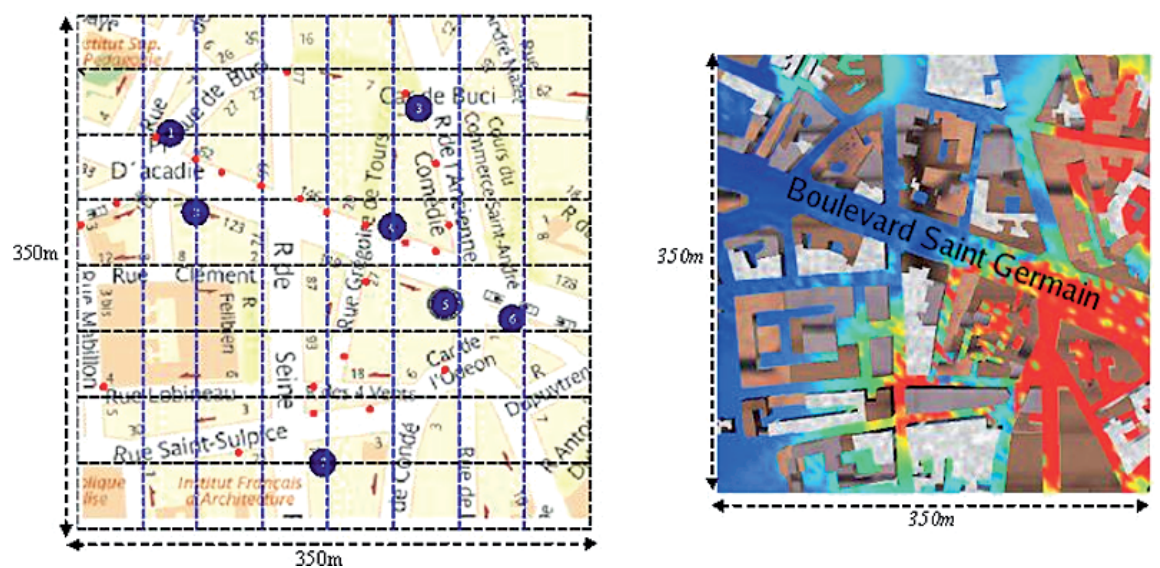

Fig. 7 The EMF Visual simulation is taking account of the position of the base stations (dots on the left) and of the reflectors (buildings)

therefore qualify the result as a guess field (like in meteorological applications (Chilès and Delfiner, 1999)).

The histograms of the data and the model output are both right-skew as they reflect/reproduce the same type of physical phenomenon. However, one cannot expect them to be perfectly compatible due to the qualitatively different sources of error,

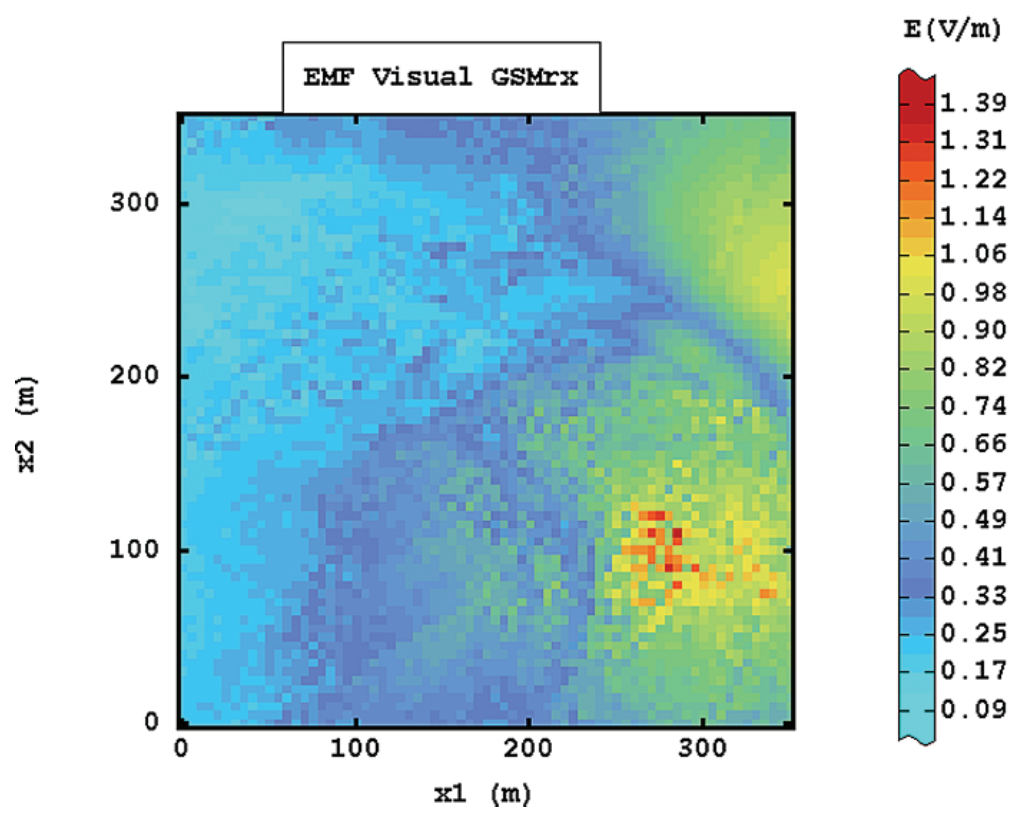

Fig. 8 Guess field generated with EMF Visual using known antenna positions and reflectors, setting likely values for their characteristics 


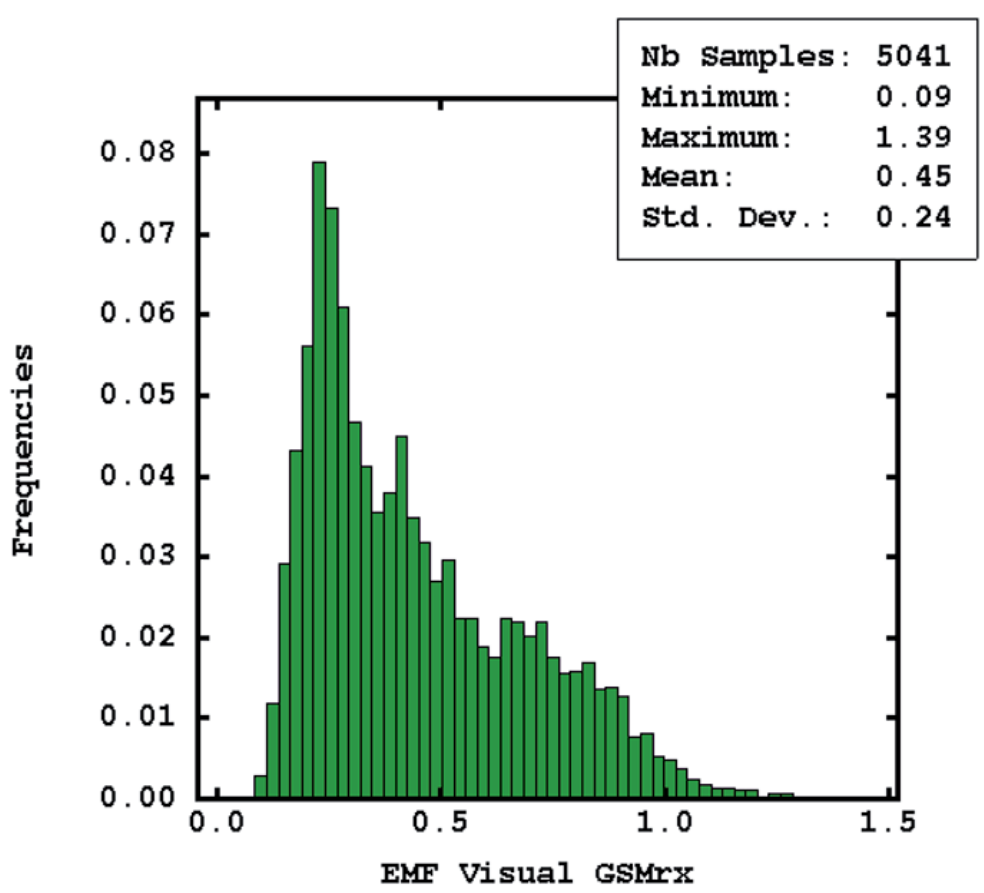

Fig. 9 Histogram of EMF Visual GSMrx values

to the non-stationarity of the field and, in particular, to the sampling design of the dosimeter data with samples arranged along lines.

The EMF Visual guess field provides an inaccurate sketch of the electromagnetic propagation from known point source locations and with known obstacles. This needs to be corrected with the help of the measurements. Conversely, the measurements do not include explicit information about the location of the sources and the reflectors, so the inclusion of the EMF Visual output is a way of bringing that information into the interpolation problem.

A balance has to be found between the data and the model output: this will result from the statistical analysis and modeling of the spatial variation of the residuals, i.e. the differences between the measurements and the numerical model output.

\section{Combining Data and Guess Field by Kriging}

The method we apply is known in geostatistics as kriging with external drift (KED) (Wackernagel, 2003; Chilès and Delfiner, 1999) and has been applied to many problems. Kriging is a spatial regression based on a statistical model of the spatial variation. The use of numerical model output as external drift is fairly recent in geostatistics (Wackernagel et al., 2004) but is common practice in meteorology and oceanography. KED is implemented in the following steps: 
1. fit the numerical model output to data by least-squares (LS-FIT),

2. compute the differences (residuals) between data values and corresponding LS-FIT values,

3. compute and model the auto-correlation of the residuals using the variogram,

4. final KED estimate based on the residuals' variogram model and the LS-FIT as external drift.

After performing the first two steps, which are elementary, we wish to characterize the spatial correlation of the residuals. This is performed in geostatistics with the variogram $\gamma(\mathbf{h})$, ie the expectation of the squared differences of pairs of values $Y$ that are $\mathbf{h}$ apart,

$$
\gamma(\mathbf{h})=\frac{1}{2} E\left[(Y(\mathbf{x}+\mathbf{h})-Y(\mathbf{x}))^{2}\right]
$$

where $\mathbf{x}$ and $\mathbf{x}+\mathbf{h}$ are two locations in geographical space. In practice, the variogram can be computed for different directions by calculating mean-squared differences of data values for pairs of points belonging to given distance and angle classes.

The variogram of GSMrx dosimeter measurements was computed into perpendicular directions of space and is displayed on Fig. 10 for the NS and EW directions.

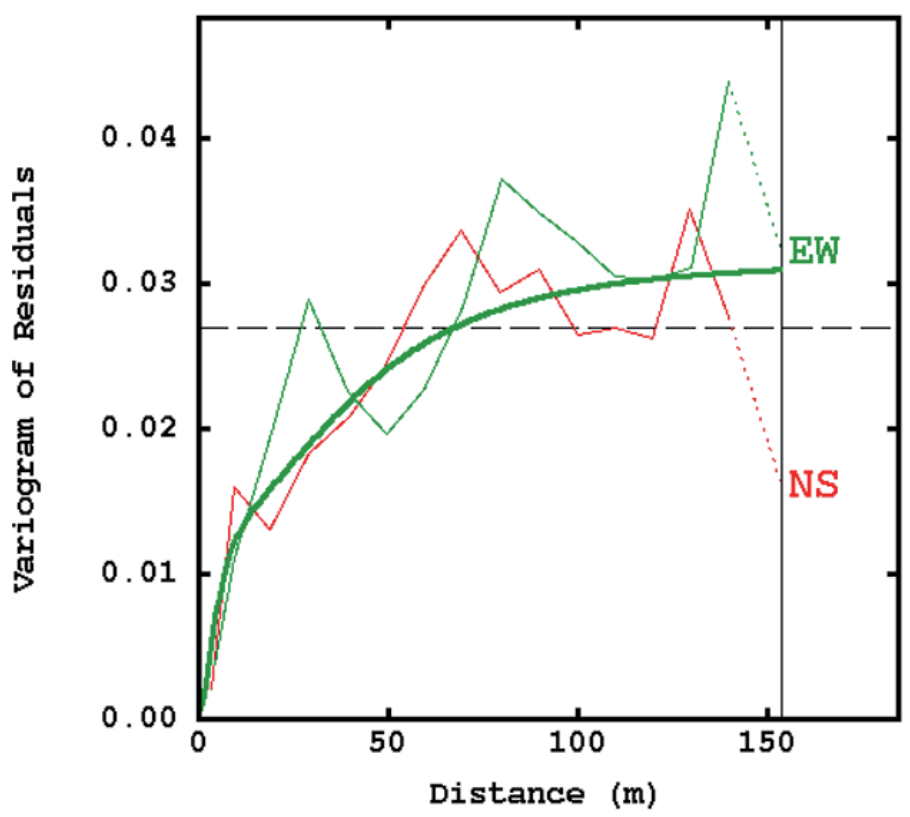

Fig. 10 Variogram of residuals between samples and EMF Visual model output. The dashed horizontal line represents the variance. The thin lines are respectively the experimental variograms computed in the North-South (NS) and East-West (EW) directions. The thick line represents the isotropic variogram model consisting of: a small measurement-variance term, a short-range $(16 \mathrm{~m})$ and a long-range $(140 \mathrm{~m})$ Cauchy-type variogram structure 


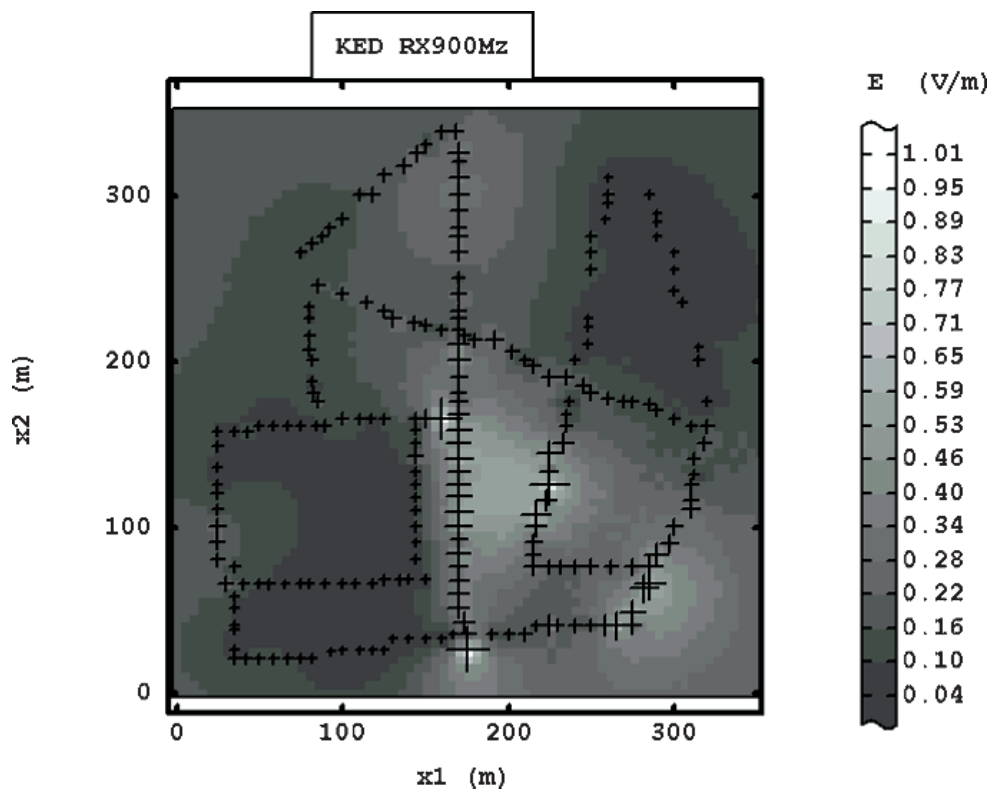

Fig. 11 Kriged map of GSMrx exposure: the "hot spots" are due to the 3 outliers

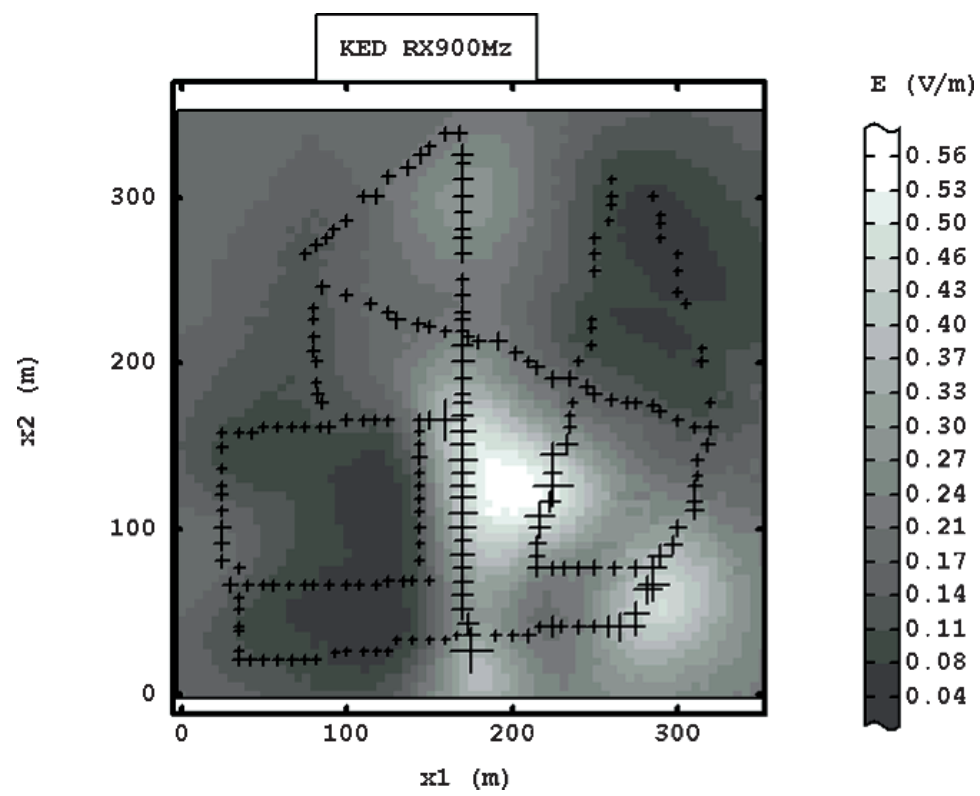

Fig. 12 Kriged map of GSMrx exposure, filtering small-scale variation: large-scale phenomena are emphasized 
The directional variograms obviously overlay, so that we could fit an isotropic variogram model. We use a nested variogram model consisting of the sum of a term corresponding to the variance of measurement-error and of two Cauchy-type variogram terms, which are commonly used in exploration geophysics to model the spatial variation of magnetic and gravimetric data (Spector and Grant, 1970; Chilès and Delfiner, 1999).

The map of the GSMrx values estimated by KED is shown on Fig. 11. The influence of the three dosimeter outliers is quite important and leads to three small-scale "hot spots" on the map, which are eventually due to fading.

As an alternative representation we propose to filter small-scale variation (see Wackernagel (2003) for details on methodology) by removing the variation associated both with the measurement variance and the short-range Cauchy-type structure. The resulting map is displayed on Fig. 12: it emphasizes large-scale phenomena and in particular the areas of higher exposure.

\section{Conclusion}

The purpose of this paper is to provide a first example of application of geostatistical methods to radio-electric exposure mapping. The methodology proposed so far is simple as it belongs to the realm of linear geostatistics.

Important aspects that have been played down in this first application are the pronounced right-skew shape of the distribution of GSMrx dosimeter values (Fig. 5), the censoring by the dosimeter of values below $0.05 \mathrm{~V} / \mathrm{m}$ and the status of outliers. These questions will need a careful treatment and will lead to the application of more sophisticated methodology.

Acknowledgments Geostatistical calculations were performed with the software package Isatis (www.geovariances.fr).

\section{References}

ANFR (2004) Panorama du rayonnement électromagnétique en France. Technical report, Agence Nationale des Fréquences, Maisons-Alfort. Available on: www.anfr.fr

Daley R (1991) Atmospheric Data Analysis. Cambridge University Press, Cambridge

De Doncker P, Dricot JM, Meys R, Hélier M, Tabbara W (2006) Electromagnetic fields estimation using spatial statistics. Electromagnetics 26:111-122

Chilès JP, Delfiner P (1999) Geostatistics: Modeling Spatial Uncertainty. Wiley, New York

ICNIRP (1998) Guidelines for limiting exposure to time-varying electric, magnetic, and electromagnetic fields (up to $300 \mathrm{GHz}$ ). Health Phys, 74(4):494-522

Larchevêque E., Dale C., Wong M-F, Wiart J. (2005) Analysis of electric field averaging for in situ radiofrequency exposure assessment. IEEE Trans on Vehicular Tech 54(4): 1245-1250

Spector A, Grant FS (1970) Statistical models for interpreting aeromagnetic data. Geophysics 35:293-302

Wackernagel H (2003) Multivariate Geostatistics: an Introduction with Applications, 3rd edn Springer-Verlag, Berlin 
Wackernagel H, Lajaunie C, Blond N, Roth C, Vautard R (2004) Geostatistical risk mapping with chemical transport model output and ozone station data. Ecological Model 179:177-185

Wiart J., Dale C., Bosisio A. V., Le Cornec A (2000) Analysis of the influence of the power control and discontinuous transmission on RF exposure with GSM mobile phones. IEEE Trans on EMC 42(4):376-385 